\title{
NIVEL DE CONOCIMIENTOS, ACTITUDES Y PRÁCTICAS SOBRE MÉTODOS ANTICONCEPTIVOS EN LOS ESTUDIANTES DE LA FACULTAD DE MEDICINA HUMANA "DANIEL ALCIDES CARRIÓN", AÑO 2017-I, ICA-PERÚ Level Of Knowledge, Attitudes And Practices On Contraceptive Methods In The Students Of Human Medicine School "Daniel Alcides Carrión" In The Year 2017-i, Ica-perú
}

Hida Adelaida Guerrero-Ortiz ${ }^{\mathrm{a}, \mathrm{b}}$, Marcia Benavides-Espinoza ${ }^{2, \mathrm{~b}}$, Elvis Kevin Guzman-Barrantes ${ }^{2, \mathrm{~b}}$, Carlos Cesar Meneses-Prieto ${ }^{2, b}$

1. Médico Gíneco-Obstetra

2. Medicina Humana

a. Dirección Regional de Salud de Ica

b. Facultad de Medicina Humana, Universidad Nacional San Luis Gonzaga de Ica

\section{Correspondencia:}

Marcia Benavides Espinoza

Domicilio: Urba. Santa María G

$\mathrm{No}_{140}$, Ica.

Telefono: 994798487

Correo Electrónico:

marcia_fresa_14@hotmail.com

Contribuciones De Autoría: GOHA, BEM, GBEK, MPCC participaron en el diseño del estudio, el análisis de los datos, revisaron críticamente el artículo y aprobaron la versión final.

Conflicto De Intereses: No declarados.

Financiamiento: Autofinanciado.

Recibido: 05-11-2017

Aceptado: 20-11-2017

Publicado: 20-12-2017

\begin{abstract}
RESUMEN:
Objetivo: Determinar el Nivel de Conocimientos, Actitudes y Prácticas sobre Métodos Anticonceptivos en los estudiantes de la Facultad de Medicina Humana "Daniel Alcides Carrión" de la Universidad San Luis Gonzaga de Ica, en el año 2017-I, Ica-Perú. Material y Metodos: El estudio es de tipo observacional, descriptivo de corte transversal. De acuerdo a los criterios de inclusión y exclusión se consideraron 163 estudiantes, los datos fueron obtenidos a través de fichas de recolección de información. Para el análisis estadístico se utilizó el Microsoft Excel versión 2013 y SPSS versión 22. Resultados: En relación a los conocimientos se encontró un $90.6 \%$ de conocimientos adecuados respecto a los preservativos, un $79.8 \%$ con respecto a la píldoras anticonceptivas; un $75.3 \%$ posee un conocimiento adecuado del método del ritmo. La relación encontrada respecto al conocimiento de un método anticonceptivo y el año de estudio fue directa. En cuanto a la edad de inicio de la actividad sexual encontramos que el promedio fue de 18 años con una edad mínima de 13 años; un $34.9 \%$ refirió ser sexualmente activo de los cuales sólo el $32 \%$ refiere haber usado un método anticonceptivo en su primera relación sexual. Un 53\% refiere que la actitud a la hora de recibir información por parte de la universidad es muy buena. De los estudiantes que refirieron no haber iniciado su vida sexual activa, un $75 \%$ refiere que su intención de uso sería el preservativo y que la cualidad por la que elegirían un método anticonceptivo sea el menor daño a su salud es un $87 \%$. Conclusiones: El nivel de conocimientos, actitudes y prácticas sobre métodos anticonceptivos en los estudiantes de medicina es bueno y se relaciona con el año de estudio.
\end{abstract}

Palabras Claves: Nivel de conocimiento, actitudes, prácticas, estudiantes, método anticonceptivo.

\section{ABSTRACT:}

Objetive: Determinate the Level of Knowledge, Attitudes and Practices on Contraceptive Methods in the students of school of Medicine "Daniel Alcides Carrión" UNICA in the year 2017-I, Ica-Perú. Material And Methods: This studio is observational, descriptive cross sectional. For the statistics analysis we used Microsoft Excel 2013 and SPSS 22 version. Results: According the age of onset of sexual activity we found that the average age was 18 years old wich $34.9 \%$ referred been sexual actives and online the $32 \%$ refers that they used a contraceptive method on their first sexual activity. A 53\% referred that the attitude about receiving information from the University was good. From the students who referred hasn't started an active sexual life a $75 \%$ referred that they intention for using a contraceptive method could be the preservative and the cuality that they crossed it is because it has a less damage to their health in a $87 \%$. Conclusions: The level of knowledge, attitude, and practices about contraceptive methods in the human medicine students it's good and it's related with the year of study.

Keywords: Level of knowledge, attitude practices, students, contraceptive methods. 


\section{INTRODUCCIÓN}

Desde la perspectiva sociológica se ha considerado a la juventud como una etapa de muchas transformaciones que operan y determinan la personalidad de los individuos. Una de estas cualidades de estos jóvenes es la promiscuidad sexual entre pares como resultado de la intensidad de relaciones sociales ligadas a la actividad educativa y de formación profesional a que están abocados. Pese a ser estudiantes universitarios y tener acceso a la información sobre salud sexual y reproductiva, estos no siempre están conscientes de la necesidad de usar métodos anticonceptivos. (1)

Los Métodos Anticonceptivos tienen la finalidad de prevenir embarazos no deseados e infecciones de transmisión sexual. Este trabajo es una alternativa para dar a conocer una sexualidad responsable en la cual la persona que acepta y pone en práctica el uso de estos es consciente de la necesidad de evitar un embarazo no deseado, enfermedades de transmisión sexual, y tener el conocimiento básico para llevar una vida sexual responsable. Es imperante actualmente para un buen desarrollo humano. (2)

La anticoncepción existe desde que se descubrió que las relaciones coitales tenían por consecuencia el embarazo; que ha ido evolucionando desde un rudimentario condón elaborado con la vejiga de un animal, hasta anticonceptivos micro-dosificados que actualmente se comercializan. El uso de anticonceptivos entre los jóvenes es irregular y poco difundido a pesar de su aumento en los últimos años. Además, cometen bastantes fallos en el uso de una contracepción adecuada, y cuanto más jóvenes son menos probable es que empleen la contracepción. Referente al uso del preservativo, la Organización Mundial de la Salud ha declarado que, para la mayor parte de jóvenes sexualmente activos, el preservativo debe ser el método anticonceptivo de primera elección con ventajas adicionales sobre otros anticonceptivos al evitar no sólo embarazos no deseados; sino también infecciones de transmisión sexual. (3)

En la actualidad se viven una serie de problemáticas sociales las cuales, según la UNESCO ha aumentado alarmantemente con los años. Dentro de dichas problemáticas se destaca de manera particular los embarazos no deseados, las enfermedades de trasmisión sexual, que según diferentes autores están dadas por la desinformación o las malas prácticas anticonceptivas. (4)

El acceso a métodos anticonceptivos eficaces y seguros que se ofrecen a la población han permitido protegerse de enfermedades de trasmisión sexual y consecuentemente ha permitido a algunas mujeres planificar su maternidad, pero el desconocimiento de estos llevan hacer mal uso o a no acceder a ellos. (5)

Actualmente, dos de los problemas de salud pública más importantes que afectan a los jóvenes, son el embarazo no deseado y las enfermedades de transmisión sexual; el uso de métodos anticonceptivos puede servir como solución para ambos. Si bien muchos de estos métodos son conocidos por los jóvenes, la mayoría no los utiliza por vergüenza de adquirirlos, y a la carencia de servicios de salud y consejería que les garanticen una privacidad y confidencialidad merecidas.

Conscientes de la problematización actual en cuanto a la salud sexual y reproductiva de los adultos jóvenes, que en su mayoría son quienes conforman el universo estudiantil de nuestra facultad, es que mostramos la necesidad de realizar un estudio con fines académicos para el cual contamos con algunos antecedentes de estudio.

En el 2011 Alvarado y colaboradores Realizaron un estudio descriptivo, cuantitativo y transversal. Obteniendo como resultados: el promedio de edad de las encuestadas es de 20.45 años ( \pm 2.90$)$ de las cuales el $99.49 \%$ afirma conocer cómo prevenir un embarazo, el $47.95 \%$ de estudiantes tiene un conocimiento adecuado sobre métodos anticonceptivos. (6)

Además, en el 2014, Pedraza y colaboradores realizo un estudio cuyo objetivo fue determinar la opinión de los estudiantes del programa de Medicina de la Universidad del Magdalena. En donde se encontró que la muestra estuvo conformada por 192 mujeres (75.6\%) y 62 varones (24.4\%), con una edad promedio de 20 años. cual mostró que la vasectomía y la ligadura de trompas eran las que más demandas presentaban, seguido por el preservativos y las pastillas. En cuanto a preferencias y/o seguridad, $14.2 \%$ de los entrevistados señaló a las pastillas o píldoras como "muy buenas"; $36.6 \%$ lo hizo frente a los condones o preservativos; $6.3 \%$ consideró los óvulos, jaleas, espumas o diafragmas; $16.9 \%$ prefiere los dispositivos intrauterinos; $13.4 \%$ optó por las hormonas inyectables. (4) Se ha encontrado también el estudio realizado por Panta en el 2016 cuyo objetivo fue determinar la relación existente entre los conocimientos y actitudes sobre el uso del anticonceptivo oral de emergencia en un grupo de estudiantes que cursaban el 5 to año de pregrado de Medicina Humana en la UNMSM. De 109 estudiantes de Medicina, el $11 \%$ (12 estudiantes) obtuvo conocimiento alto, el $62.3 \%$ (68 estudiantes) obtuvo nivel de conocimiento medio y el $26.6 \%$ (29 estudiantes) obtuvo nivel de conocimiento bajo sobre la anticoncepción oral de emergencia En cuanto a las Actitudes, el 14.6\%(16 estudiantes) de los encuestados presentó actitud de aceptación, el 70.6\%(77 estudiantes) presentó actitud indecisa y el $14.6 \%$ (16 estudiantes) presentó actitud de rechazo (...): En términos generales los estudiantes de 5 to año de Medicina Humana (89\%) presenta conocimiento entre bajo y medio sobre la anticoncepción oral de emergencia. La actitud general de los estudiantes de 5 to año de medicina es indecisa sobre la anticoncepción oral de emergencia. (7)

Viendo estos estudios podemos darnos cuenta de la necesidad de realizar un estudio similiar que englobe los conocimientos actitudes y practicas sobre el uso de métodos anticonceptivos.

Con lo anteriormente expuesto, el presente estudio de investigación considera conveniente responder a la interrogante: ¿CUÁL ES EL NIVEL DE CONOCIMIENTOS, ACTITUDES Y PRÁCTICAS SOBRE MÉTODOS ANTICONCEPTIVOS DE LOS ESTUDIANTES DE LA FACULTAD DE MEDICINA HUMANA "DANIEL ALCIDES CARRIÓN" AÑO 2017 - I, ICA-PERÚ?

Este trabajo beneficiará en primer lugar a los estudiantes de la Facultad de Medicina Humana "Daniel Alcides Carrión", quienes después de la investigación y de acuerdo con los resultados se podrá establecer un diagnóstico situacional acerca de los conocimientos, actitudes y prácticas sobre métodos anticonceptivos, y de esta manera servirá como precedente para estudios futuros y mejorías del nivel encontrado en el presente. Se beneficiarán también los estudiantes de otras facultades; ya que siendo nuestra facultad formadora en Ciencias de la Salud, siendo los indicados para impartir conocimientos, impartir actitudes y promover la práctica del uso de métodos anticonceptivos; recomendaremos la realización de talleres educativos; los cuales homogenicen un nivel óptimo en todos los estudiantes de nuestra Universidad.

\section{MATERIAL Y MÉTODOS}

Se trata de un estudio de tipo Observacional Descriptivo de corte transversal; al no haber sido influenciadas en sus respuestas, las mismas que han sido observadas en los estudiantes de la Facultad de Medicina Humana "Daniel Alcides Carrión" de la Universidad Nacional San Luis Gonzaga de Ica.

El diseño es descriptivo, dado que describe el nivel de Conocimientos, Actitudes y Prácticas sobre métodos anticonceptivos en los estudiantes de la Facultad de Medicina Humana "Daniel Alcides Carrión" de la Universidad Nacional San Luis Gonzaga de Ica.

Se tomó como población a los estudiantes de la Facultad de Medicina Humana "Daniel Alcides Carrión" de la Universidad Nacional San Luis Gonzaga de Ica, matriculados en el año académico 2017-I, que tiene una población de 480 estudiantes, los cuales fueron obtenidos usando la lista oficial de estudiantes del $1^{\circ}$ a $6^{\circ}$ año, proporcionado por el departamento académico de la facultad. La muestra está constituida por 163 estudiantes de la 
Facultad de Medicina Humana "Daniel Alcides Carrión" de la Universidad Nacional San Luis Gonzaga de Ica, matriculados en el año académico 2017-I

Para la toma de información se coordinó con los estudiantes en sus horarios disponibles, solicitándoles nos brinden 20 minutos de su tiempo para el llenado del instrumento, logrando la mayoría de encuestas. Asimismo, se encuestó a estudiantes individualmente siguiendo la misma temática descrita anteriormente; obteniéndose así el número necesario de encuestas para completar la muestra calculada. Previo a ello se les brindó la información respecto a los investigadores, asesor de la investigación, institución a la que representamos, título de la investigación y la especificación de salvaguardar su identidad en todo momento; así como el derecho de retirarse de la investigación si así lo desearan. Además requerimos de la aceptación del consentimiento informado por parte de los estudiantes para su participación.

Cuando manifestaron haber llenado íntegramente el instrumento, realizamos una revisión para corroborarlo; caso contrario solicitamos que completara la información. Al culminar la recolección de datos, revisamos y posteriormente eliminamos aquellas fichas que se encontraron incompletas.

Se utilizó como instrumento un adoptado y adaptado de la tesis "Actitudes, Conocimientos y Prácticas en la población adolescentes sobre el uso de Anticonceptivos en la provincia de Ica, 2008". (3)

En esta ficha de recolección de datos, se adaptaron los cuestionarios redirigiéndolos hacia adultos jóvenes, que en su mayoría forman parte de la población de nuestra facultad. El instrumento cuenta con un consentimiento informado, en donde se encuentra información explícita acerca del estudio. Además cuenta con 5 ítems referentes a aspectos personales, 7 ítems de aspecto familiar y religioso, 4 ítems de aspecto psicosocial y 32 preguntas dirigidas a los conocimientos, actitudes y prácticas sobre métodos anticonceptivos.

El cuestionario consta de un total de 48 preguntas en las cuales 32 son dirigidas a los conocimientos, actitudes y prácticas sobre métodos anticonceptivos. De los cuales 15 de ellas son referentes a Conocimientos, 10 de Actitudes y 7 de Prácticas; cuyos intervalos están definidos según la escala de Stanones de la Curva de Gauss. Además 12 preguntas son de índole general y sociodemográfico; de las cuales se obtendrá una relación cruzada para un mejor análisis estadístico correlativo.

Después se realizó un análisis univariado (frecuencia, media y porcentaje) y Bivariado ( $\left(\mathrm{Chi} \rrbracket \wedge_{2}\right.$ ); determinándose un valor " $p$ " menor de 0,05 como estadísticamente significativo. Se elaboraron gráficos haciendo uso de los programas Microsoft Excel y SPS 22.0

\section{RESULTADOS}

En nuestra investigación se tomó como muestra los estudiantes de la Facultad de Medicina Humana "Daniel Alcides Carrión" de la UNICA, en total participaron 163 estudiantes de los diferentes años; por tanto fueron clasificados por grupos de acuerdo a los años de estudia que cursaban. Los grupos no fueron homogéneos debido a que estudiaban diferente número de alumnos en cada año.

Se encuestaron a un total de 163 personas. La edad media es de 20 años, una edad mínima de 17 años y una edad máxima de 26 años. Del marco poblacional, el $47 \%$ ( 76 alumnos) es del sexo femenino y el 53\% (87 alumnos) del sexo masculino, siendo el 99\% (161 alumnos) solteros y solo $1 \%$ (02 alumnos) es conviviente.

Del estado civil de los padres, el 78\% (de 127 alumnos) son casados y el 22\% (36 alumnos) manifiestan que están separados, de ellos, el 3\% (05 personas) si madre cuenta con un trabajo, el 17\% (28 personas) tiene a su padre con trabajo y un $80 \%$ tiene ambos padre trabajando. Las madres de los alumnos en un 3\% (04 madres) tiene educación primaria, el 47\% (77 madres) tienen educación secundaria y el 50\% (82 madres) tienen educación superior, y de los padres de los alumnos, menos de 1\% (01 padres) tiene educación primaria, 36\% tiene educación secundaria, y un 64\% (104 padres) tiene educación superior.
De los alumnos, según su año académico, el 29\% son del primer año, $25 \%$ del segundo año, 31\% del tercer año, $8 \%$ del cuarto año, $6 \%$ del quinto año y $2 \%$ del sexto año (Gráfico $\mathrm{N}^{\circ} 1$ ), siendo el $70 \%$ de los alumnos de la ciudad de Ica.

\section{Año de estudios}

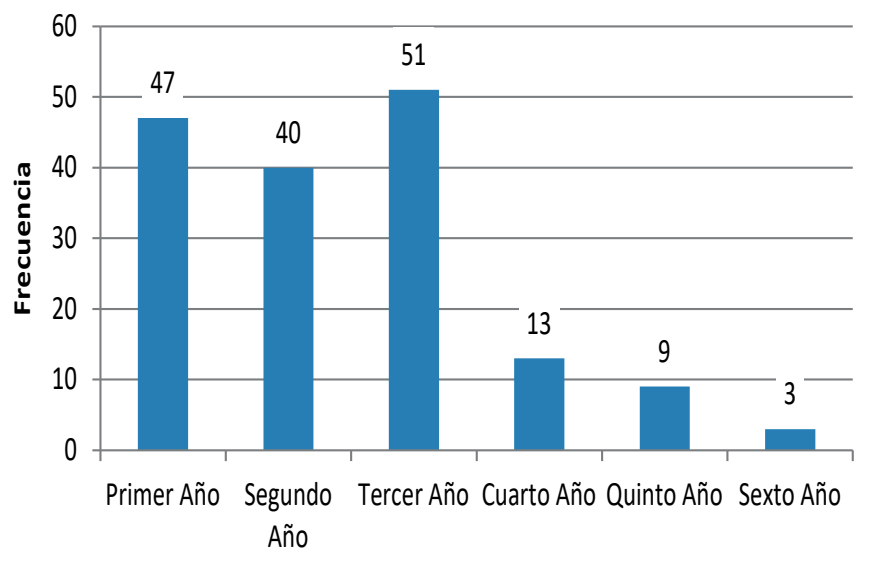

\section{Año de estudios}

Gráfico 1: Año de estudios que cursan los estudiantes de medicina de la facultad “daniel alcides carrión” de la única, 2017

En la muestra de estudio se encontró que sólo el 1\% de los estudiantes no conoce ningún método anticonceptivo. De los que manifestaron conocer entre 1, 23 a más métodos anticonceptivos, el $100 \%$ manifestó que el preservativo es el MAC más conocido; y en segundo lugar es la Píldora del día siguiente con un 85\%. (Gráfico $\mathrm{N}^{\circ} 2$ ).

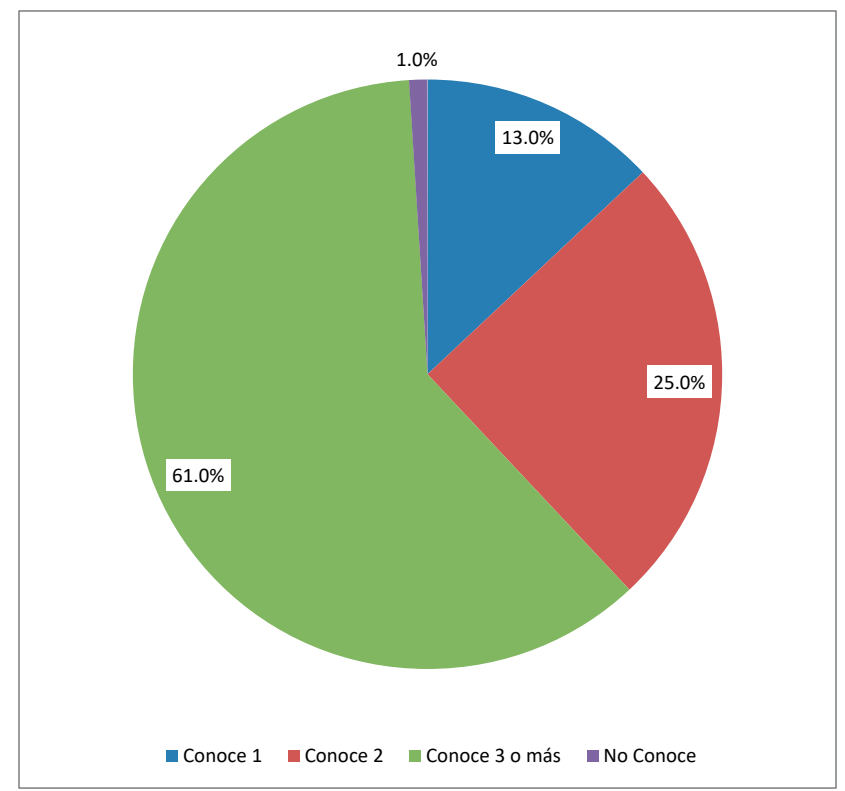

Gráfico 2: Conocimientos sobre métodos anticonceptivos de los estudiantes de medicina de la facultad "daniel alcides carrión" de la única, 2017

En relación a los conocimientos sobre Métodos Anticonceptivos, destaca que el $100 \%$ de entrevistados conoce que el preservativo no se debe lavar para volver a usarlo; el $98.2 \%$ sabe que el preservativo protege de las ITS. En cuanto a las Píldoras anticonceptivas, resalta que el $93.3 \%$ sabe que previamente requiere evaluación médica respectiva. Con respecto al método del Ritmo, $85.9 \%$ de los encuestados conoce que durante la menstruación no existen posibilidades de embarazo. Finalmente, un $78.5 \%$ conoce que la píldora del día siguiente se puede tomar máximo hasta $72 \mathrm{hrs}$ posterior a la relación sexual. (Tabla $\mathrm{N}^{\circ} 1$ ) 
Tabla $\mathrm{n}^{\circ}$ 1: Conocimientos sobre métodos anticonceptivos según tipo de mac en estudiantes de medicina de la facultad "daniel alcides carrión" de la única, 2017

\begin{tabular}{|c|c|c|}
\hline Conocimiento sobre MAC & $\mathbf{n}$ & $\%$ \\
\hline \multicolumn{3}{|l|}{ Preservativo } \\
\hline $\begin{array}{l}\text { No se lava el preservativo para usarlo } \\
\text { nuevamente }\end{array}$ & 163 & $100.00 \%$ \\
\hline $\begin{array}{l}\text { El preservativo no se puede llevar a } \\
\text { cualquier lugar }\end{array}$ & 120 & $73.60 \%$ \\
\hline El preservativo protege de las ITS & 160 & $98.20 \%$ \\
\hline \multicolumn{3}{|l|}{ Píldoras Anticonceptivas } \\
\hline $\begin{array}{l}\text { No son eficaces si se toman antes de cada } \\
\text { RS }\end{array}$ & 90 & $55.20 \%$ \\
\hline Requiere de previa evaluación médica & 152 & $93.30 \%$ \\
\hline Tomarlas no protege de ITS & 148 & $90.80 \%$ \\
\hline \multicolumn{3}{|l|}{ Método del Ritmo } \\
\hline $\begin{array}{l}\text { Durante la menstruación no hay posibilidad } \\
\text { de embarazo }\end{array}$ & 140 & $85.90 \%$ \\
\hline $\begin{array}{l}\text { Mujeres con menstruación irregular tienen } \\
\text { riesgo de embarazo }\end{array}$ & 125 & $76.70 \%$ \\
\hline $\begin{array}{l}\text { A mitad del ciclo menstrual es más probable } \\
\text { el embarazo }\end{array}$ & 103 & $63.20 \%$ \\
\hline Píldora del Día Siguiente & & \\
\hline $\begin{array}{l}\text { Se puede tomar hasta } 72 \text { hrs después de la } \\
\text { relación sexual }\end{array}$ & 128 & $78.50 \%$ \\
\hline
\end{tabular}

Con respecto al año de estudios de los alumnos, no se encuentra asociación significativa con el nivel de conocimiento (p-valor: o.809), se muestra que con mayor frecuencia en conocimientos malos, lo tienen los año primero, tercero y quinto, los de sexto año no presentaron malos conocimientos (Tabla No2 2 .

Tabla $\mathbf{n}^{\circ}$ 2: Relación entre el nivel de conocimientos y año académico de los estudiantes de medicina de la facultad "daniel alcides carrión" de la única, 2017

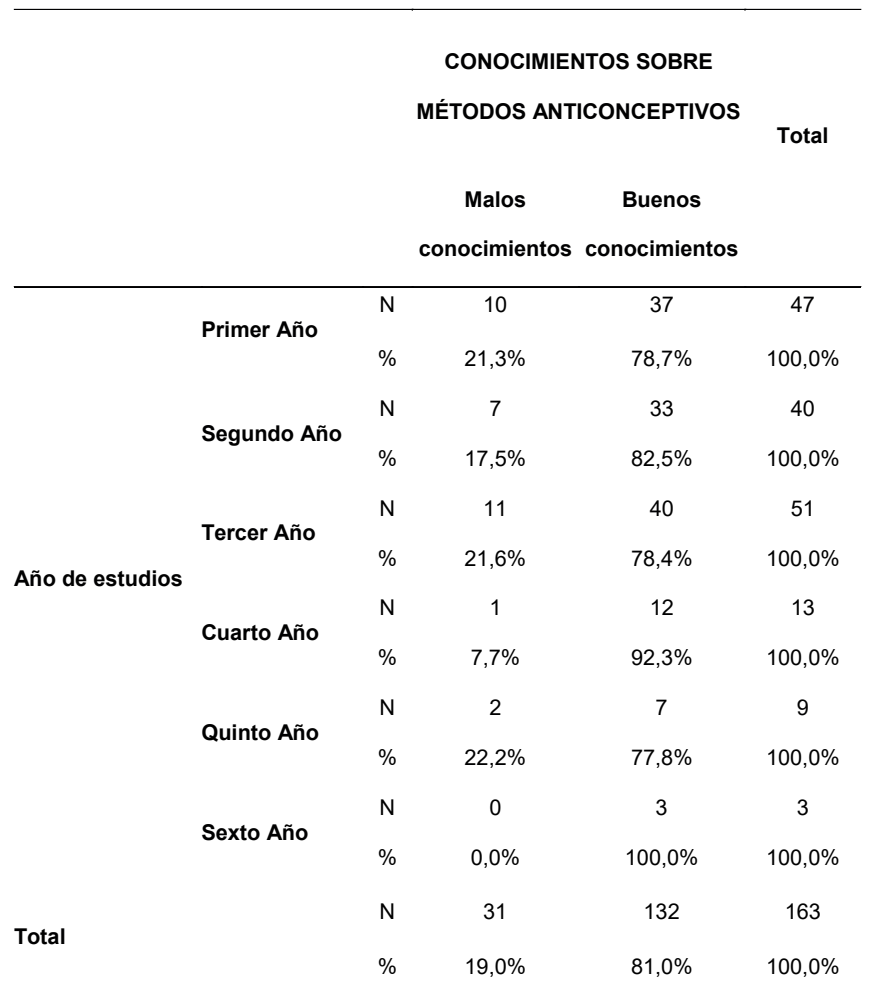

Se ha encontrado que respecto a la valoración de información obtenida por su propia cuenta la actitud es muy buena en un $43 \%$, además de que la valoración de la información obtenida por la Universidad es muy buena en un $53 \%$. Siendo además relevante que la valoración de los conocimientos sobre métodos anticonceptivos son buenos en un $55 \%$.(Grafico $\mathrm{N}^{\circ} 3$ ).

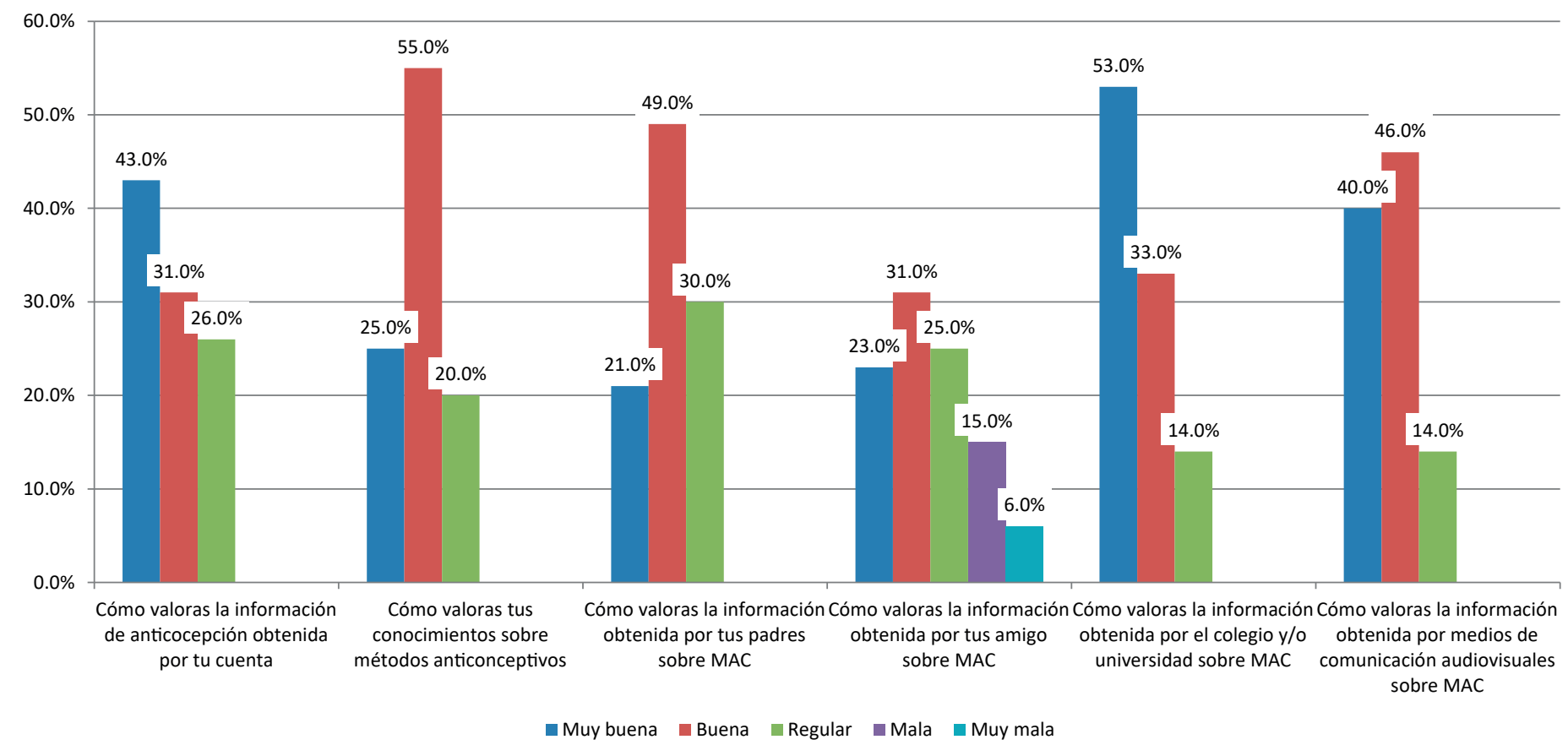

Gráfico 3: Actitudes frente al conocimiento de métodos anticonceptivos en los estudiantes de medicina de la facultad "daniel alcides carrión" de la única, 2017 
Un 60\% de nuestros encuestados refieren que la $1^{\circ}$ fuente a la que acudirías en busca de información sería un personal de salud, en contraste con un $33 \%$ quien refiere casi nunca acudiría a sus padres en busca de información sobre MAC.

De los alumnos que empezaron su vida sexual, el $87 \%$ (24 alumnos) siguen usando métodos anticonceptivos, del 13\% (05 alumnos) que no usa algún método anticonceptivo, el $20 \%$ refiere que es por un costo elevado, baja satisfacción sexual y por ser dañino para la salud respectivamente, mientras que el $40 \%$ tiene otro motivo por la cual ya no mantiene el uso de los métodos anticonceptivos.

En información por la que nunca uso un método anticonceptivo, el 77\% (13 alumnos) mencionaron porque fue algo inesperado, el 18\% (03 alumnos) porque pensaban que no quedarían con embarazo, el 6\% (01 alumno) menciona que fue la pareja quien uso el método anticonceptivo. (Grafico $\mathrm{N}^{\circ} 4$ )

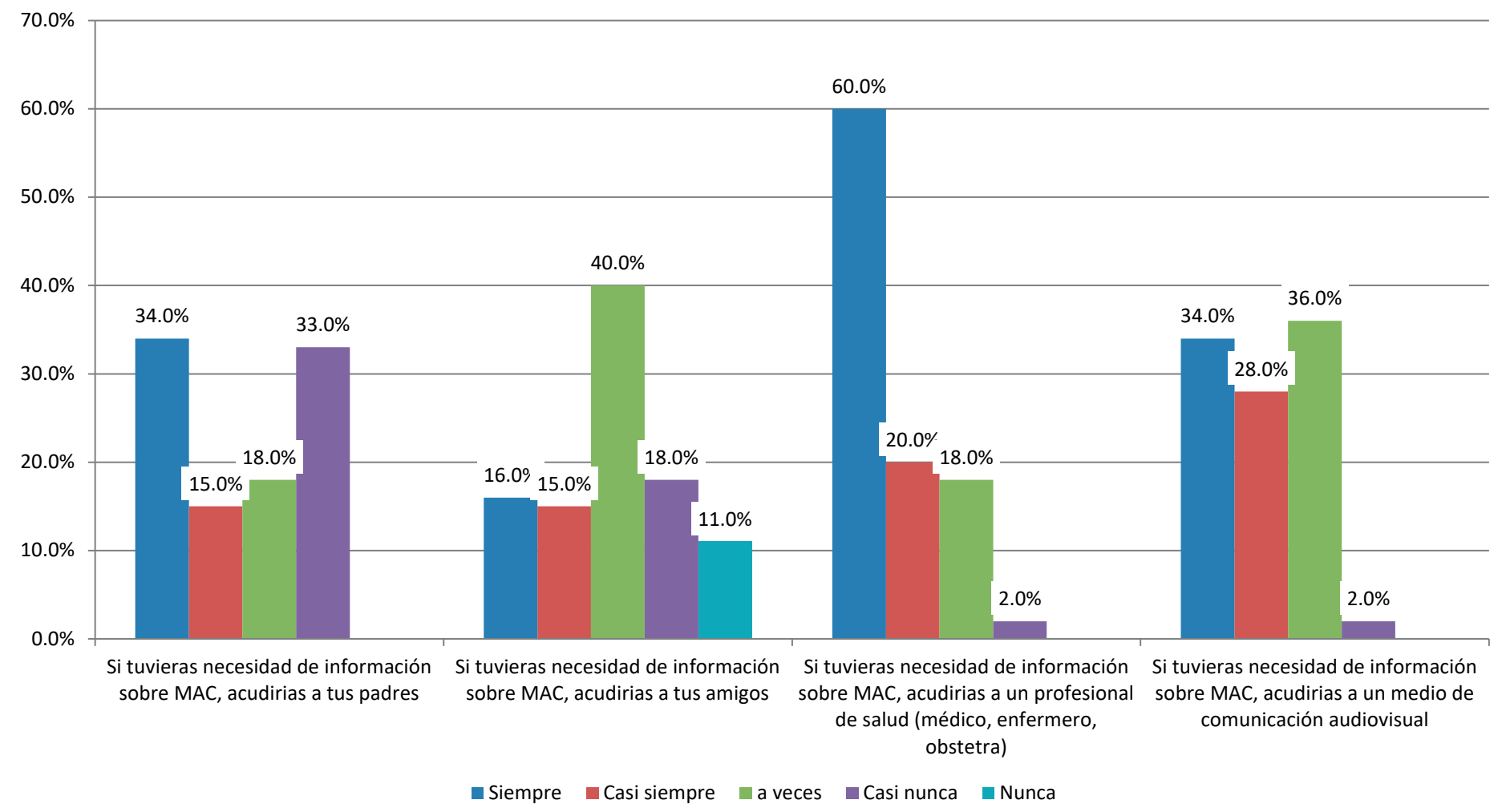

Gráfico 4: Actitud respecto a la necesidad de información en los estudiantes de medicina de la facultad “daniel alcides carrión” de la única, 2017

El método anticonceptivo que usa actualmente, en menos del $1 \%$ usa el coito interrumpido, método de DIU, píldora anticonceptiva de emergencia, mientras que el $91 \%$ ( 52 alumnos) utilizan el preservativo, de ellos, refiere que el preservativo tiene mayor frecuencia de su uso frente a los demás métodos anticonceptivos. (Grafico $\mathrm{N}^{\circ} 4$ ). Un 75\% de los encuestados refiere la primera opción sobre uso métodos anticonceptivos es el preservativo y en segundo lugar la píldora del día siguiente; ya sea este por un menor daño a su salud, bajo costo o fácil uso. (Grafico $\mathrm{N}^{\circ} 5$ )
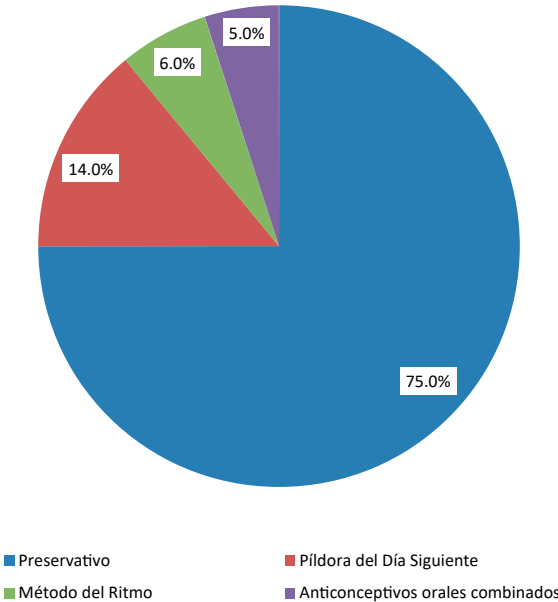

Gráfico 5: Intensión de uso de métodos anticonceptivo en la primera relación sexual en los estudiantes de medicina de la Facultad “daniel alcides carrión” de la única, 2017

\section{DISCUSIÓN}

En la muestra que obtuvimos de los estudiantes de Medicina, se encontró que la edad media de los participantes se encontraba entre los 20 años de edad, en similitud con otros estudios realizados en Nicaragua y Perú cuyas medias de edad fueron de 19 y 21 años de edad, respectivamente. $(1,5)$

Con respecto al género, se encontró que la mayoría de los participantes fueron del sexo masculino representado por un $53.4 \%$; en contraparte con otros estudios en donde el sexo femenino era el de mayor proporción $(8,5)$

En cuanto al conocimiento de los métodos anticonceptivos, se encontró que un $99 \%$ conoce la existencia de al menos un método anticonceptivo, muy similar a otros estudios realizados en universidades del Perú y Nicaragua $(9,10)$. Además de obtener un alto porcentaje de respuestas correctas referente a los métodos anticonceptivos especificados.

Por otro lado, las preguntas planteadas para cada método anticonceptivo estudiado, se encontró que el mayor porcentaje de conocimiento está en el uso del preservativo, en comparación con un estudio realizado en Santander, en donde se obtuvo un 63\% (11); por otro lado en una investigación realizada en México se encontró un porcentaje similar a lo descrito previamente (12)

Hemos encontrado que aproximadamente el $35 \%$ de los encuestados han iniciado la actividad sexual activa, de los cuales la edad promedio de inicio fue de 18 años, cuya límite inferior de edad encontrada fue de 13 años, en el estudio realizado en la universidad de San Martín de Porres Perú se encontró que el 54.7\% presentó actividad sexual entre los 12 y 15 años de edad (13). Del mismo modo, en otro estudio realizado en Cuba se obtuvo que el $84 \%$ de 
los encuestados inició su actividad sexual entre los 12 y 15 años de edad. (14); lo que nos muestra que menor edad de inicio de actividad sexual, no muestra mayores diferencias respecto a nuestro trabajo; sin embargo el porcentaje de personas que han iniciado su actividad sexual es menor respecto a estos dos estudios. Lo que nos indica que la primera relación sexual ocurre cada vez en etapas más tempranas de la vida, lo cual se asocia a un mayor riesgo de embarazos no deseados, Infecciones de trasmisión sexual y otras patologías relacionadas.

Los conocimientos en relación al año académico presentan un relación directa respecto a que a mayor año académico cursado por el estudiante, mayor será el conocimiento sobre métodos anticonceptivos, lo cual se traduce que conforme un estudiante avanza académicamente adquiere más conocimientos. Además hemos encontrado que a partir del zer año de educación universitaria, los conocimientos se tornan mayores, probablemente porque a partir de estos se llevan asignaturas específicas respecto a métodos anticonceptivos (11).

Con respecto al nivel de conocimiento encontrado en relación al género obtuvimos que el mayor porcentaje de conocimiento era bueno en cuanto al género femenino como masculino, siendo estos un $81.6 \%$ y $80.5 \%$ respectivamente. En un estudio realizado en Cuba se obtuvo que el mayor porcentaje de conocimiento estuvo representado por el sexo femenino (15); asimismo en un estudio realizado en México se encontró un $90 \%$ de las mujeres señaló tener conocimiento sobre los MAC en comparación con los varones, siendo un $70 \%$ aquellos que desconocían su utilización y funcionamiento (11)

Con respecto a las actitudes que muestran nuestros encuestados, encontramos que más del 50\% refiere que valora positivamente la información obtenida por su cuenta y la Universidad. En un estudio realizado por Barragan y colaboradores se encontró que existe una relación directa entre la percepción del conocimiento y la información brindada por los padres. (16)

Respecto a la necesidad de obtener información encontramos que un $60 \%$ de nuestros encuestados acudirían siempre a un médico y/u otro profesional; en cambio en el estudio realizado que lleva por título "Incidencia de la educación, núcleo familiar y sociedad en el nivel de conocimiento de métodos anticonceptivos", se encontró que los encuestados acudirían a su centro de estudios en búsqueda de información. (16)

En relación a las prácticas del uso de métodos anticonceptivos, un $68 \%$ de los encuestados sexualmente activos refiere que no usó algunos métodos anticonceptivos en su primera relación sexual, ya sea por tratarse de una situación inesperada, falta de confianza, pérdida de romanticismo. En un estudio realizado en Colombia se encontró que un $70 \%$ de mujeres y un $89 \%$ de varones hicieron uso de métodos anticonceptivos en su primera relación sexual. El principal método anticonceptivo utilizado fue el preservativo masculino (14). Además en un estudio realizado en Colombia respecto al uso de preservativo en la primera relación sexual se encontró que un $56 \%$ de los jóvenes usó el preservativo como método anticonceptivo en su primera relación sexual (17). Además en otro estudio realizado en universitarios se obtuvo que el método anticonceptivo utilizado con mayor frecuencia en su primera relación sexual fueron los de barrera (18).

Por otro lado, respecto a la cualidad del método anticonceptivo para su uso encontramos que la mayor acogida de este fue de causar menor daño a la salud representado por un $92 \%$; en contraparte en un estudio realizado en Bolivia los estudiantes refirieron que usarían el preservativo por su función de prevenir ITS y su cualidad de ofrecer seguridad para evitar embarazos no deseados (19). 


\section{BIBLIOGRAFÍA}

1. Urrutia A, Torres M, Vallejos N, Bonilla P. Riesgos que corren los estudiantes de II y III año de la carrera de Trabajo Social de la UNAN León, ante el inadecuado uso de anticonceptivos. [Monografía para obtener licenciatura en Trabajo Social]. 2014. [Citado el o9 de Febrero del 2017]. Universidad Nacional Autónoma de Nicaragua, UNAN - León Facultad De Ciencias de La Educación y Humanidades. Departamento de Trabajo Social. Disponible desde: http://riul.unanleon.edu.ni:8080/jspui/bitstream/123456789/4260/1/ 228683.pdf

2. Pelaez, J. Importancia del Conocimiento y Promoción de la Anticoncepción de Emergencia. Rev Cubana Obstet Ginecol [online]. 2011, vol.37, n.3, pp. 399-408. ISSN 0138-600X.[Citado el 17 de Marzo del 2017]. Disponible en: scielo.sld.cu/scielo.php?script=sci_arttext\&pid=S0138-600X201100 0300012

3. Chanco A, Lazarte R. Actitudes, Conocimientos y Prácticas en la población adolescentes sobre el uso de Anticonceptivos en la provincia de Ica, 2008.[Tesis para obtener título de médico cirujano]. Universidad San Luis Gonzaga de Ica. Perú 2008.

4. Pedraza L, et al. Opiniones hacia las prácticas anticonceptivas en estudiantes de la Universidad del Magdalena, Santa Marta (Colombia). Rev Ciencia y Salud Virtual. [online]. 2014. [Citado el 17 de Marzo del 2017]. 6(2):pp. 82-91. Disponible desde: http://revistas.curnvirtual.edu.co/index.php/cienciaysalud/article/vi ew/418

5. Fonseca M, Macedo H, Tapullima L. Factores demográficos y Culturales Relacionados al uso de Métodos Anticonceptivos en Estudiantes de la Facultad de Enfermería UNAP, Iquitos 2015. [Tesis para obtener título profesional de Enfermería]. Perú. 2015. [Citado el 17 de Marzo del 2017]. Disponible en: http://repositorio.unapiquitos.edu.pe/bitstream/handle/UNAP/332 3/Minor_Tesis_Titulo_2016.pdf?sequence=1\&isAllowed=y

6. Alvarado,A. Idrovo, M. Manzano, P. Conocimientos, Actitudes y Prácticas sobre Prevención de Embarazo en las Estudiantes de la escuela de Medicina de la Facultad de Ciencias Médicas de la Universidad de Cuenca, año 2010. Ecuador-2011. [Citado el 16 de Marzo del 2017]. Disponible en: http://dspace.ucuenca.edu.ec/bitstream/123456789/3456/1/MED91. pdf

7. Panta, M. Conocimiento y Actitudes sobre anticoncepción oral de emergencia en estudiantes de 5 to año de Medicina Humana de la Universidad Nacional Mayor de San Marcos. [Tesis Doctoral]. UNMSM. Lima 2016. [citado el 10 de Febrero del 2017].Disponible e http://cybertesis.unmsm.edu.pe/bitstream/cybertesis/4749/1/Panta _om.pdf

8. Yubero $M$, Larrañaga $E$, Yubero S. Actitudes y comportamiento sexual de riesgo de embarazo en jóvenes universitarios. Rev Psic Criança e do Adolescente. [Online]. 2013. [Citado el 15 de Marzo del 2017]; 4(2): pp 11-29. Disponible en http://repositorio.ulusiada.pt/bitstream/11067/752/1/rpca_4_2_1.pdf 9. Méjico J, Melgar F, Mendoza A, Salinas W. Nivel de Conocimiento sobre Métodos Anticonceptivos entre Estudiantes de Medicina Humana de $1^{\circ}$ a $3^{\circ}$ año. Perú. Rev Horiz Med. [Online] 2012, Jul-Set. [Citado el 16 de Marzo del 2017]. 12(3): pp 25-41. Disponible en: http://www.horizontemedicina.usmp.edu.pe/index.php/horizonte med/article/view/129/127

10. Calero O, Cardoza S, Pilarte R. Conocimientos, Actitudes y Prácticas sobre el uso de Métodos anticonceptivos en estudiantes de I año de medicina de la Facultad de Ciencias Médicas, UNAN-Managua, Mayo 2015.[Tesis doctoral]. Nicaragua. Universidad Nacional Autónoma de Nicaragua, Managua Facultad de Ciencias Médicas UNAN. 2016

11. Atehortua W, Acosta S, López A, Suárez J, Melo A, Carrascal S. Conocimiento Métodos anticonceptivos en estudiantes de pregrado. Rev Ciencia y Salud. [Online]. 2011. [Citado el 16 de Marzo del 2017]. 8(1): pp 48-54. Disponible en: http://respuestas.ufps.edu.co/ojs/index.php/cienciaycuidado/articl e/view/473/490

12. Solano C, Romero L, Castañeda D], Ortega S, García Y. Percepción de los estudiantes de Medicina sobre el uso de Métodos Anticonceptivos, ICSa 2016. Mexico. Universidad Autónoma del Estado de Hidalgo. 9(5). 2016 Disponible en: https://repository.uaeh.edu.mx/revistas/index.php/ICSA/article/vie $\mathrm{w} / 2260 / 2258$

13. Navarro M, López A. Nivel de Conocimiento y Actitudes sexuales en adolescentes de la urbanización Las Palmeras - Distrito de Morales. Periodo Junio- Setiembre 2012. [Tesis para obtener Título profesional de Obstetra]. Tarapoto-Perú. Universidad Nacional de San Martin Facultad de Ciencias de la Salud Escuela Académica Profesional de Obstetricia. 2012

14. Covarrubias E. Ramírez R. Verde E, Rivas J, Rivero L. Utilización de Métodos Anticonceptivos en estudiantes de Enfermería. Colombia. Investig Enferm. Imagen Desarr. 2016; 18(2): 31-43. http://revistas.javeriana.edu.co/index.php/imagenydesarrollo/articl e/view/10298/12219

15. Rodríguez A, Sanabria G, Contreras M, Perdomo B. Estrategia educativa sobre promoción en Salud Sexual y reproductiva para adolescentes y jóvenes universitarios. Rev Cubana Salud Pública. [Online] 2013, Cuba. [Citado el 16 de Marzo del 2017]. 39(1): pp 161-174. Disponible en: http://scielo.sld.cu/pdf/rcsp/v39n1/spu15113.pdf

16. Barragán C, Beltrán M, Díaz D, Murcia S, Ramírez D, Rojas N, Rubio P. Incidencia de la educación, núcleo familiar y sociedad en el nivel de conocimiento de los métodos anticonceptivos. Colombia-Bogotá. Universidad Antonio Nariño Facultad de Ciencias de la Educación. 7(14): pp. 65-76. 2016

17. Uribe I, Bahamón M, Reyes R, Trejos AM, Alarcón Y. Percepción de autoeficacia, asertividad sexual y uso del condón en jóvenes colombianos. Rev Acta Colombiana Psicología.[Online] 2016, Bolivia-Potosí. [Citado el 17 de Marzo del 2017]. 1(20): pp 204-211. Disponible en: http://editorial.ucatolica.edu.co/ojsucatolica/revistas_ucatolica/ind ex.php/acta-colombiana-psicologia/article/view/1079/1232

18. Mondragón L, Arteaga R, Islas G, Sánchez H. Conocimientos, Actitudes y Prácticas sobre los Métodos Anticonceptivos en estudiantes de Medicina. Rev Med "Dr. José Sierra Flores". [Online]. 2014, Dic. [Citado el 17 de Marzo del 2017]. 2(28): pp 71-80. Disponible http://www.une.edu.mx/Resources/RevistaMedicina/2014/2014-02/ 3-Conocimientos,\%2oactitudes\%20y\%2opr\%C3\%A1cticas\%20sobre\%20 los\%20m\%C3\%Agtodos\%20anticonceptivos\%20en\%2oestudiantes $\% 20$ de\%2omedicina.pdf

19. Ávalos L, Briceño D, Gonzáles MG, Paita D, Rico V, Saldaña AM. Factores que influyen en la utilización de métodos anticonceptivos en los jóvenes. Rev Per Obst Enf. [Online]. 2010. [Citado el 17 de Marzo]. 6(2): pp 88-96. Disponible en: http://www.aulavirtualusmp.pe/ojs/index.php/rpoe/article/view/65 $3 / 507$ 